\title{
,awe \\ Gender Differences in Environmental Correlates of Cycling Activity among Older Urban Adults
}

\author{
Lanjing Wang ${ }^{1}$, Xiayidan Xiaohelaiti ${ }^{1}$, Yi Zhang ${ }^{1, *}$, Xiaofei Liu ${ }^{2}$, Xumei Chen ${ }^{2}$, Chaoyang Li ${ }^{1}$, Tao Wang ${ }^{1}$ (D) \\ and Jiani $\mathrm{Wu}^{1}$
}

Citation: Wang, L.; Xiaohelaiti, X.; Zhang, Y.; Liu, X.; Chen, X.; Li, C.; Wang, T.; Wu, J. Gender Differences in Environmental Correlates of Cycling Activity among Older Urban Adults. Land 2022, 11, 52. https:// doi.org/10.3390/land11010052

Academic Editors: Vanessa

Zorrilla-Muñoz, Eduardo Fernandez, Blanca Criado Quesada, Sonia De Lucas Santos, Jesus Cuadrado Rojo and Maria Silveria Agulló-Tomás

Received: 29 November 2021

Accepted: 29 December 2021

Published: 30 December 2021

Publisher's Note: MDPI stays neutral with regard to jurisdictional claims in published maps and institutional affiliations.

Copyright: (C) 2021 by the authors. Licensee MDPI, Basel, Switzerland. This article is an open access article distributed under the terms and conditions of the Creative Commons Attribution (CC BY) license (https:// creativecommons.org/licenses/by/ $4.0 /)$.
1 State Key Laboratory of Ocean Engineering, China Institute for Urban Governance, Shanghai Jiao Tong University, Shanghai 200240, China; lanjing.wang@sjtu.edu.cn (L.W.); xayda.x@sjtu.edu.cn (X.X.); cyljjf@sjtu.edu.cn (C.L.); wangtao127@sjtu.edu.cn (T.W.); JiaNiZi@sjtu.edu.cn (J.W.)

2 Key Laboratory of Advanced Public Transportation Science, China Academy of Transportation Sciences, MOT, Beijing 100029, China; liuxf@motcats.ac.cn (X.L.); chenxm@motcats.ac.cn (X.C.)

* Correspondence: darrenzhy@sjtu.edu.cn

\begin{abstract}
Cycling is a form of active transport that can improve the level of health among the elderly population. However, little is known about the environmental correlates of bicycle use among older adults. This study investigated the relationship between the built and social environment and the gender differences in cycling frequency among older urban adults in China. The data were derived from a household travel survey in 2012 and covered thirty-three urban neighborhoods in Zhongshan. The results suggest that denser intersections are negatively related to cycling trips among both older men and women. Reverse associations for either gender, however, are observed between the average income in a neighborhood and cycling frequency. For older women, living far from a bus stop is positively correlated to an increase in daily cycling trips. For older men, social environment, including the proportions of employed or elderly people in a neighborhood, is significantly associated with cycling activity. The findings facilitate the understanding of the gender gap in cycling activity among older urban adults, and help towards designing effective planning strategies as health interventions.
\end{abstract}

Keywords: gender differences; built environment; social environment; cycling activity; older urban adults

\section{Introduction}

The elderly population is the fastest-growing group worldwide [1]. The world is expected to experience a significant demographic shift over the next few decades. By 2050, the world's elderly population ( 65 years and older) is estimated to reach 1.5 billion, a drastic growth from 727 million in 2020 [2]. Between 2020 and 2050, the proportion of the global older adult population will nearly double from $9.3 \%$ to $16 \%$ [3]. By $2050,80 \%$ of older adults will be living in low- and middle-income countries. This raises major challenges to ensure that both health and social systems are ready to face this demographic shift [3].

Research findings indicate that active transport provides significant health benefits to the elderly population [4]. As a crucial form of active transport, cycling helps to prevent chronic diseases and bone fractures in older adults [5]. A recent review suggests that the health benefits of cycling outweigh the risks [6]. Older adults who choose cycling over driving for short trips have the most statistically significant estimated gain in life, compared to other age subgroups [6]. Specifically, for older women, a small amount of daily bicycling reduces the risk of atrial fibrillation and is beneficial for muscle strength and functional abilities $[7,8]$. Therefore, cycling may be a viable option for older adults where a favorable policy exists and a bicycle-friendly social and built environment is created [9].

Considering the benefits, policies have been implemented to enhance bicycle use among the general population. For example, in the Netherlands and Denmark, the cycling transportation infrastructure is sufficient and the modal choice of cycling is high, indicating 
the potential of bicycle use among older individuals [10]. However, few policies targeting the elderly population can be found, possibly due to the risks (exposure to traffic and air pollution) and physical barriers preventing older adults from cycling. The environmental correlates of cycling activity have been revealed among the general population. Nevertheless, the potential of cycling among older adults has been neglected for research and practice [11]. The social and built environment may facilitate or restrict older adults' participation in cycling activity [1]. There was limited evidence revealing the factors significantly associated with cycling activity among older adults [12]. Furthermore, older women partake in less bicycle use than their male counterparts. However, few studies have investigated the gender differences in the effects of the built and social environment on bicycle use among older adults.

Which environmental features are associated with cycling activity among older urban adults? Are there any gender differences in the environment-cycling activity relationships? Which planning strategies offer the most promising effects for increasing cycling among older urban men and women? This study attempts to answer these questions by investigating the gender-specific environmental correlates of bicycle use among older urban adults. Using data from thirty-three urban neighborhoods in Zhongshan, China, we investigated the environmental correlates of older urban adults' daily cycling trips for transportation and recreation, controlling for attitudinal and sociodemographic attributes. The findings will facilitate planners' and policymakers' attempts to increase bicycle use among both older urban men and women by optimizing land use planning and improving environmental conditions.

This paper is organized as follows: Section 2 reviews relevant literature. Section 3 details the data and method. Section 4 presents findings from negative binomial regression. Section 5 includes discussion and policy implications, and Section 6 concludes with limitations and strengths.

\section{Literature Review}

The planning [13-15] and public health [16-18] fields have mutually contributed to "environment-active transport" studies, with a focus on older adults. However, most research emphasized walking, with very little on cycling $[19,20]$. Generally, previous studies categorized the environmental factors as two types: The built environment and the social environment [21].

The built environment is defined as "the human-made space in which people live, work, and recreate on a day-to-day basis" [22] (p. 24) and "encompasses places and spaces created or modified by people including buildings, parks, and transportation systems" [23] (p. 1446). Prior studies have examined the built environment factors relating to older adults' cycling in different contexts [24-27]. However, few studies have explored the associations among the aging population in developing countries. The built environment factors commonly utilized are defined as the "five Ds": density, design, diversity, distance to transit, and destination accessibility [28]. A denser population is related to a higher propensity to cycle among older adults in China [29]. However, the urban density shows negativity in explaining bicycle use among older adults in the Netherlands [30]. Welldesigned cycling infrastructure has shown positive effects, as expected [25,31-34]. Mixed development is linked to an increase in cycling frequency among older adults $[24,25,29,31,35]$. Being adjacent to services and destinations is also attributed to a higher propensity to cycle $[25,26,36,37]$. Inadequate transit services are related to more cycling trips, as older adults may opt to cycle for medium-distance trips $[25,35,38]$.

The social environment "includes the culture that the individual was educated or lives in, and the people and institutions with whom they interact" [39] (p. 465). Role models and neighborhood social cohesion were associated with walking duration, and peer support may facilitate physical activity among older adults [21,22]. The role models in previous studies included both the young and the old who favor active transport most. Another study in Zhongshan, China, found that when the proportion of the elderly population 
exceeds 15\% in a neighborhood, bus use among older adults decreases [40]. In denser areas with a higher ratio of younger adults, young travelers may choose the bus as part of an active lifestyle. They will act as role models for older adults. Therefore, in areas with a higher ratio of older people, the effects of role models will decrease, leading to a decrease in the modal share of bus use. However, little is known about the social environment correlates of older adults' cycling activity. A negative association was observed between the proportion of the aged in a community and cycling among rural older adults in China [25]. Factors related to the social image of cycling also influenced the cycling among older adults [9].

Gender-specific differences between environmental factors and physical activity among older adults have been preliminarily examined. However, most studies focused on walking. Women were less active than men, and environmental factors were significantly related to women's physical activity and walking [41]. Self-efficacy, density, and design were related to older men's walking, and self-efficacy and destinations were related to older women [42].

Most "environment-active transport" research has been conducted in developed countries. The findings are sometimes not transferable to developing countries with ultra-high population densities, including China [18]. Recently, scholars have begun to explore how environmental variables are related to active transport and health promotion among different age groups [43]. Nonetheless, little research has been carried out to explore the gender-specific environment correlates of cycling frequency among older urban adults throughout the world. The cycling activity of older urban adults is essential for efficacious interventions on health promotion. This study addresses these gaps by specifically examining the built and social environment correlates of bicycle use among older urban men or women in Zhongshan, China. In this paper, "cycling" includes utilitarian and recreational trips. Older adults focus on the group aged above 59.

It is worth noting the dataset and modeling approach in the present study are the same as in our previous study [44]. However, the two studies are significantly different regarding research focus and contribution. Our previous study [44] focused on the general older urban population and employed only built environment variables. It is among the earliest attempts to explore the environmental correlates of cycling among older adults [44]. This time we concentrated on the gender differences in the influences of both built and social environment on cycling trips of older urban adults. The findings will help the design of gender-specific interventions to facilitate cycling among older urban men and women and contribute to the land use-travel literature.

\section{Data and Methods}

\subsection{Study Area}

In China, the elderly population is defined as adults aged over 59, according to the Law of the People's Republic of China on Protection of the Rights and Interests of the Elderly. By 2020, the elderly Chinese population was over 200 million, reaching nearly $15 \%$ of the general population. It is necessary to encourage active transport among older Chinese adults as a health intervention. In 2007, the "China Healthy Lifestyle for All" initiative launched a campaign of "Ten Thousand Steps a Day" [45], aiming to promote walking among the general population. Cycling was once a prevailing travel mode in China; however, the modal split of cycling has decreased due to rapid motorization [46]. The factors facilitating cycling among Chinese older adults have barely been studied, and few interventions have been initiated to revive bicycle use in China. We chose Zhongshan to disentangle the gender-specific cycling activity among older urban adults in China [47,48]. Zhongshan is a medium-sized city in Guangdong Province, China (Figure 1). In China's urban agglomerations, there are over two dozen cities with similar urban transport patterns to Zhongshan [49]. Therefore, the findings of the study may transfer to those cities. 


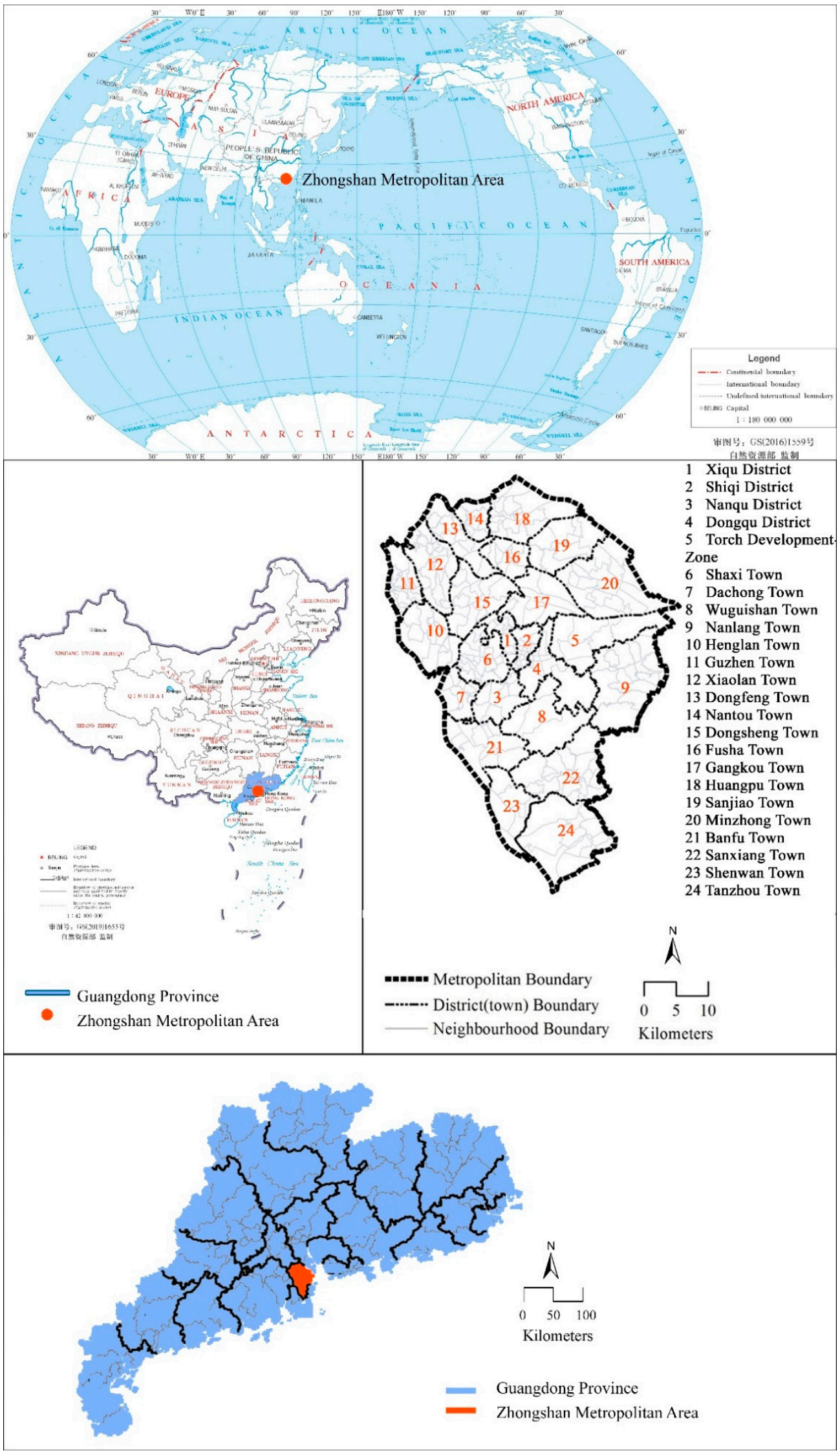

Figure 1. Study area. 


\subsection{Data Collection}

The Zhongshan Household Travel Survey (ZHTS) in 2012 provided the bicycle use data [50]. The survey covered the entire Zhongshan Metropolitan Area. The survey included a self-reported one-day travel diary and the sociodemographics of the urban adults. The sampling method used was stratified random sampling. The sample size was 616 older urban women and 648 men from thirty-three neighborhoods. The sample rate was $2.0 \%$.

The built and social environment data were provided by Zhongshan Municipal Bureau of Urban Planning in 2012 [47,48]. We imported the data into ArcGIS for analysis. The data included: (1) neighborhood boundaries and neighborhood-level socio-demographics (population, dwelling units, and employment); (2) five types of land uses (residential land, commercial land, industrial land, green space land, and other land); (3) road networks; and (4) bus stops.

\subsection{Characterization of Social Environment and Built Environment Variables}

The environmental variables were characterized based on neighborhood level [51]. Based on the administrative divisions of Zhongshan, thirty-three urban neighborhoods were selected for analysis. We characterized three neighborhood-level social environment variables, i.e., the proportions of the employed, the ratios of the aged, and the average household income.

We characterized five built environment variables according to the "five Ds" [52]. The dwelling unit density represents Density; the intersection density denotes Design; the distance between home and the closest bus stop defines Distance to Transit; the distance to the CBD stands for Destination Accessibility; and the land use mixture demonstrates Diversity [52]. The first four variables are self-explanatory. The land use mixture denotes the degree to which different land uses in a neighborhood are mixed. The land use mixture was calculated by the Entropy Index (EI) [53], wherein 0 denotes a single-use environment and 1 represents the equalization of different land uses in area coverage. $E I$ is expressed by:

$$
E I=\sum_{i=1}^{n} P_{i} \log \left(1 / P_{i}\right)
$$

where $n=$ number of different land uses, $n \geq 1 ; P i=$ percentage of land use $i$ 's coverage in the entire land use coverage. In the Chinese standard, the officially recommended proportion of residential, industrial, commercial, green space, and other types of land use is around 2:2:1:1:1 [54], generating an EI of 0.67. This proportion is employed in land use planning practices of Chinese cities, including Zhongshan. Therefore, each of the original $E I$ of a neighborhood in this study is transformed into a criterion that 0.67 is the standard 1 , and all other indexes are ranged between 0 and 1 based on the standard 1 [47].

\subsection{Modeling Approach}

Older urban adults' cycling trips are non-negative count-dependent variables. Considering the statistical characteristics of the cycling trips (Appendix A), we first chose negative binomial regression. We then applied a Vuong model selection test, and the result preferred a standard one over a zero-inflated one (Figure 2). Finally, we utilized a negative binomial regression model to examine the gender-specific sociodemographics and the attitudinal and environmental correlates of bicycle use among older urban adults. The multicollinearity of all the independent variables were checked by the variance inflation factor (VIF). None of the VIFs were more significant than ten, suggesting a low degree of multicollinearity. 


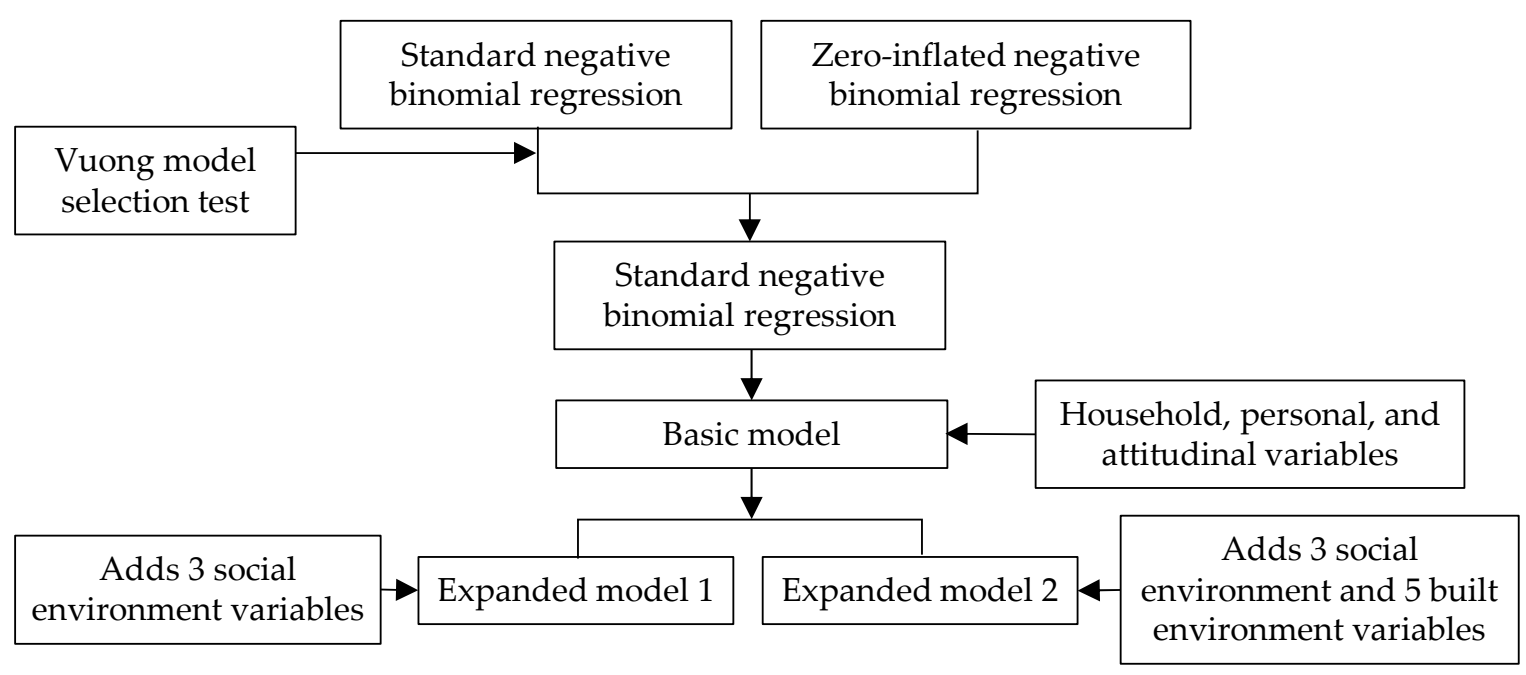

Figure 2. The modeling approach.

We built models separately for older men and women and used the same independent variable sets. We rewrote the negative binomial regression model with natural log transformation for calibrating the coefficient with Stata 12.0. The basic model was as follow:

$$
\begin{gathered}
N_{f r}=\beta_{0}+\beta_{1} \times \text { SIZE_1 }+\beta_{2} \times \text { SIZE_ } 2+\beta_{3} \times \text { EMPLOYED }+\beta_{4} \times \text { H_HIGHINC }+ \\
\beta_{5} \times \text { H_MEDINC }+\beta_{6} \times \text { AGE }+\beta_{7} \times \text { P_HIGHINC }+\beta_{8} \times \text { P_MEDINC }+ \\
\beta_{9} \times \text { FAV_BIKE }+\beta_{10} \times \text { FAV_WALK }+\beta_{11} \times \text { FAV_EBIKE }+\beta_{12} \times \text { FAV_BUS }
\end{gathered}
$$

where $N f r=$ frequency (times/day) of cycling of an older urban adult; SIZE_1 and SIZE_2 = dummies for the household size of one and two (with a household size of more than two as a reference category); EMPLOYED = number of family members employed; H_HIGHINC and H_MEDINC = dummies for high household monthly income over 6000 Chinese Yuan (Renminbi) (RMB, 6.4 Renminbi $\approx 1$ US Dollar) and medium between 2500 and $6000 \mathrm{RMB}$ (with a reference category of 0-2500 RMB); AGE = respondent's age in years; P_HIGHINC and P_MEDINC = dummies for high personal monthly income over 3000 RMB and medium between 1200 and 3000 RMB (with 0-1200 RMB as a reference category), FAV_BIKE, FAV_WALK, FAV_EBIKE, or FAV_BUS = whether the respondent's favorite travel mode is bicycle, walking, e-bike, or bus.

The regression proceeded in two expanded models based on the basic model (Figure 2). The expanded model 1 adds three neighborhood-level social environment attributes as independent variables, where N_EMPLOY and N_AGED demonstrate the proportions of the employed and the aged in a neighborhood, respectively; N_AVGINC represents the average monthly income of a neighborhood. The expanded Model 2 includes five built environment variables, in which DWELL_DEN, INTER_DEN, DIST_BUS, DIST_CBD, and LAND_MIX denote dwelling unit density, intersection density, the distance between home and the closest bus stop, Euclidean distance from the centroid of the neighborhood to the CBD, and land use mixture. Among the five attributes, DIST_CBD is a household-level attribute, and the other four are neighborhood-level.

\section{Results}

\subsection{Descriptive Statistics}

In Zhongshan, older urban men and women make 3.06 and 2.84 trips per day, respectively, among which 0.37 (men) and 0.18 (women) trips were cycling (Tables 1 and A1) [55]. One in five older urban adults lives alone, and $40 \%$ live with one partner. One-fifth live in high-income households. About $16 \%$ of males preferred cycling to other modes, whereas that figure was only $9 \%$ for females. The respondents' average age was 67 . The social 
and built environment of both men and women was very similar due to the random sampling method.

Table 1. Description of variables.

\begin{tabular}{|c|c|c|}
\hline Category & Variable & Description \\
\hline Dependent Variable & Frequency & Daily Cycling Trips, Count \\
\hline \multirow{7}{*}{ Household variables } & SIZE_1 & One member in a household, binary, $1=$ yes \\
\hline & SIZE_2 & Two members in a household, binary, $1=$ yes \\
\hline & SIZE_2+ & Three or more members in a household, binary, $1=$ yes \\
\hline & EMPLOYED & Number of household members employed, count \\
\hline & H_HIGHINC & High household income (>6000 RMB/month), binary, 1 = yes \\
\hline & H_MEDINC & Medium household income (2500-6000 RMB/month), binary, $1=$ yes \\
\hline & H_LOWINC & Low household income ( $<2500 \mathrm{RMB} /$ year), binary, 1 = yes \\
\hline \multirow{4}{*}{ Personal variables } & AGE & Age in years, count \\
\hline & P_HIGHINC & High personal income (>3000 RMB/month), binary, $1=$ yes \\
\hline & P_MEDINC & Medium personal income (1200-3000 RMB/month), binary, 1 = yes \\
\hline & P_LOWINC & Low personal income ( $<1200 \mathrm{RMB} /$ month $)$, binary, 1 = yes \\
\hline \multirow{5}{*}{ Attitudinal variables } & FAV_BIKE & The respondent's favorite travel mode is bicycle, binary, $1=$ yes \\
\hline & FAV_WALK & The respondent's favorite travel mode is walking, binary, $1=$ yes \\
\hline & FAV_EBIKE & The respondent's favorite travel mode is e-bike, binary, $1=$ yes \\
\hline & FAV_BUS & The respondent's favorite travel mode is bus, binary, $1=$ yes \\
\hline & FAV_CAR & The respondent's favorite travel mode is car, binary, $1=$ yes \\
\hline \multirow{3}{*}{$\begin{array}{c}\text { Social environment } \\
\text { variables }\end{array}$} & N_EMPLOY & Proportions of the employed in a neighborhood, continuous \\
\hline & N_AGED & Proportions of the aged in a neighborhood, continuous \\
\hline & N_AVGINC & Average monthly income of a neighborhood (in $100 \mathrm{RMB}$ ), continuous \\
\hline \multirow{5}{*}{$\begin{array}{l}\text { Built environment } \\
\text { variables }\end{array}$} & DWELL_DEN & Dwelling units' density, 1000 dwelling units $/ \mathrm{km}^{2}$, continuous \\
\hline & INTER_DEN & Intersection density, five intersections $/ \mathrm{km}^{2}$, continuous \\
\hline & DIST_BUS & Distance between home and the closest bus stop, $\mathrm{km}$, continuous \\
\hline & DIST_CBD & Euclidean distance from the neighborhood centroid to the CBD, in $\mathrm{km}$, continuous \\
\hline & LAND_MIX & Entropy Index of land use mixture, continuous \\
\hline
\end{tabular}

\subsection{Analysis of Cycling Frequency among Older Urban Men and Women}

Generally, the directions of the coefficients for sociodemographics and attitudes persisted across all models, and the coefficients indicated slight to moderate variation. The LR chi2 and Log-likelihood demonstrates the overall goodness of fit. The variations of pseudoR2, LR chi2, and Log-likelihood in expanded models suggested that the environmental variables strengthened the explanatory power and predictability of the models (Table 2).

Two personal attributes (medium personal income and favoring bicycling over other modes), one social environment attribute (average neighborhood income), and one built environment (intersection density) attribute were significantly related to both males and females. The male respondents who were pro-bicycle would have 4.37 times more cycling trips than those who were not, while the number was even more prominent for female respondents, at 7.65 times. Regarding social environments, a 100 RMB increase in average neighborhood monthly income was related to 7.35\% more cycling trips for older urban men, but $15.51 \%$ fewer for women. The cycling trips decreased by $7.39 \%$ (for men) or $18.88 \%$ (for women) when the intersection density increased by one unit, which is 5 intersections per $\mathrm{km}^{2}$. 
Table 2. Negative binomial regression analysis of cycling frequency among older urban men and women in Zhongshan.

\begin{tabular}{|c|c|c|c|c|c|c|}
\hline \multirow{3}{*}{ Variable } & \multicolumn{2}{|c|}{ Basic Model } & \multicolumn{2}{|c|}{ Expanded Model 1} & \multicolumn{2}{|c|}{ Expanded Model 2} \\
\hline & \multicolumn{2}{|c|}{ Coef. } & \multicolumn{2}{|c|}{ Coef. } & \multicolumn{2}{|c|}{ Coef. } \\
\hline & Men & Women & Men & Women & Men & Women \\
\hline \multicolumn{7}{|c|}{ Household socio-demographics (SIZE > 2 and H_LOWINC are reference categories) } \\
\hline SIZE_1 & -0.023 & $-1.273^{* *}$ & -0.110 & $-1.050 * * *$ & -0.041 & $-0.793 * * *$ \\
\hline SIZE_2 & 0.282 & $-1.853 *$ & 0.191 & $-1.409 *$ & 0.205 & $-1.463 *$ \\
\hline EMPLOYED & -0.039 & $-1.195 *$ & -0.016 & $-1.136 *$ & -0.054 & $-1.258 *$ \\
\hline H_HIGHINC & $0.751 * *$ & 0.943 & $0.840 * *$ & 1.193 & $0.790^{* *}$ & 1.260 \\
\hline H_MEDINC & 0.262 & 0.347 & 0.305 & 0.321 & 0.295 & 0.372 \\
\hline \multicolumn{7}{|c|}{ Personal socio-demographics (P_LOWINC is a reference category) } \\
\hline AGE & $-0.023^{* * *}$ & -0.034 & $-0.024^{* * *}$ & -0.044 & $-0.027^{* * *}$ & -0.037 \\
\hline P_HIGHINC & $-0.896^{* *}$ & 0.413 & $-1.110^{* *}$ & 0.696 & $-1.124^{* *}$ & 1.204 \\
\hline P_MEDINC & $-0.835 *$ & $-1.039 * *$ & $-0.992 *$ & $-0.919 * *$ & $-0.991 *$ & $-0.594^{* * *}$ \\
\hline \multicolumn{7}{|c|}{ Attitudes (FAV_CAR is a reference category) } \\
\hline FAV_BIKE & $1.681 *$ & $2.157 *$ & $1.739 *$ & $2.030 *$ & $1.817^{*}$ & $2.103 *$ \\
\hline FAV_WALK & -0.291 & -0.315 & -0.208 & -0.362 & -0.139 & -0.251 \\
\hline FAV_EBIKE & -0.347 & 0.496 & -0.272 & 0.419 & -0.258 & 0.047 \\
\hline FAV_BUS & $-2.403 *$ & -0.903 & $-2.281 *$ & -0.731 & $-2.192 *$ & -0.507 \\
\hline \multicolumn{7}{|c|}{ Social environment } \\
\hline N_EMPLOY & & & 0.308 * & -1.032 & $0.586^{* *}$ & -0.700 \\
\hline N_AGED & & & $-0.385^{* *}$ & -1.756 & $-0.658^{* * *}$ & -1.720 \\
\hline N_AVGINC & & & $0.071^{* *}$ & $-0.168 *$ & $0.087 *$ & $-0.071^{* * *}$ \\
\hline \multicolumn{7}{|c|}{ Built environment } \\
\hline DWELL_DEN & & & & & 0.024 & -0.005 \\
\hline INTER_DEN & & & & & $-0.077^{* * *}$ & $-0.209^{* * *}$ \\
\hline DIST_BUS & & & & & -0.254 & $1.083^{* * *}$ \\
\hline DIST_CBD & & & & & $-0.215^{* * *}$ & 0.017 \\
\hline LAND_MIX & & & & & -0.545 & -1.483 \\
\hline \multicolumn{7}{|c|}{ Summary statistics } \\
\hline _cons & -0.382 & 0.869 & -5.264 & 5.690 & -4.654 & 1.290 \\
\hline Number of obs. & 648 & 616 & 648 & 616 & 648 & 616 \\
\hline LR chi2 & 283.63 & 128.07 & 296.46 & 138.89 & 302.97 & 148.38 \\
\hline Prob > chi2 & 0.0000 & 0.0000 & 0.0000 & 0.0000 & 0.0000 & 0.0000 \\
\hline Pseudo-R2 & 0.2306 & 0.2470 & 0.2411 & 0.2679 & 0.2464 & 0.2861 \\
\hline Log-likelihood & -473.0756 & -195.2382 & -466.6582 & -189.8262 & -463.4049 & -185.0829 \\
\hline
\end{tabular}

Seven attributes were significantly associated solely with older urban men's cycling activity, covering all four categories. At a household and personal level, household or personal income, age, and a positive attitude towards public transportation showed significance at $90 \%$ confidence. Being one year older was related to a $2.26 \%$ reduction in cycling trips. Male respondents with high or medium incomes had $59.17 \%$ or $56.62 \%$ fewer cycling trips, respectively, than those with low incomes. Older urban men who preferred the bus to other modes made $90.96 \%$ fewer cycling trips. Regarding social environment variables, male respondents living in a neighborhood with the highest proportion of employed or aged people made $11.86 \%$ more or $8.04 \%$ fewer cycling trips, respectively, compared to those residing in areas with the lowest proportions. The only built environment attribute significantly related to male respondents was the distance to the CBD. As the results 
showed, living one kilometer farther from the CBD was associated with 19.35\% fewer cycling trips.

Four attributes were significantly associated solely with older urban women's cycling activity, among which three were related to household and one to built environment. Living alone or with a partner, female respondents made $72.01 \%$ or $84.32 \%$ fewer cycling trips, respectively, compared to those with more household members. Having one more family member employed was related to a $69.73 \%$ reduction in cycling trips. At the built environment level, older urban women make $19.53 \%$ more cycling trips if the closest bus stop is one hundred meters farther from home.

\section{Discussion and Policy Implications}

\subsection{Discussion}

One built environment variable, intersection density, is significantly related to bicycle use among both older urban men and women. In a neighborhood with denser intersections, both men and women make significantly fewer cycling trips. To be specific, the negative association of intersection density with female respondents' cycling trips was much stronger. The reasons for this finding are complex. On the one hand, this may be due to safety concerns, as a recent study suggested that cyclists are exposed to a higher risk of accidents when crossing intersections [56]. Another study in Italy, however, observed a decreased risk of pedestrian accidents at road intersections, as both pedestrians and drivers pay more attention when approaching the street nodes. [57] On another hand, denser intersections are positively related to a higher traffic density, represented by more vehicles on the roads $[33,34]$. The safety issues related to the conflicts of bicycles with other modes, especially vehicles, may increase the perceived barriers towards cycling activity among older adults [32]. The average monthly income of the neighborhood, as a social environment variable, shows reverse impacts on older urban men and women. Residing in a more affluent neighborhood, older urban men make more cycling trips, while older urban women make fewer. The underlying reasons for the results call for further study. As expected, favoring cycling over other modes is associated with an increase in bicycle use among both male and female respondents. The results indicate the potential of disseminating a healthy lifestyle regarding cycling among older urban adults.

The attributes related solely to male respondents include household and personal characteristics, the social environment, and the built environment. Being older, richer, or favoring the bus over cycling is linked to fewer cycle trips. Living in a more dynamic social environment with more employed people and fewer older people is related to an increase in cycling trips for older urban men. The results are probably connected to social norms concerning active transport [58]. Previous research has observed positive relationships between social promoters, such as having neighbors that bicycle and greater physical activity [33]. In this study, physically active older urban men may opt to reside in communities with younger or employed neighbors. Presumably, the younger or employed population tend to choose more active travel modes, including cycling. As role models, they may influence older adults to cycle as a part of an active lifestyle. However, the indepth reasons require future study. The findings indicate that forming neighborhoods with relatively balanced age or employment structures may facilitate cycling among older urban men. The built environment variable regarding commercial accessibility demonstrated a significant association. Better accessibility to the CBD was related to more bicycle use. Presumably, older urban men will opt to cycle for short-to-medium commercial trips if they live adjacent to the CBD [59]. This finding is in line with existing literature that suggests that access to destinations appeared to be important for promoting cycling among older adults [24].

The attributes related solely to female respondents included household characteristics and the built environment. For example, household size is positively associated with older urban women's cycling activity. Living with two or more family members was linked to more cycling trips. The distance between home and the closest bus stop is the only 
significant built environment factor for older urban women. Specifically, living farther from a bus stop is correlated with an increase in cycling trips. Presumably, when the closest bus stop is beyond walking distance from home, older urban women may choose cycling to access the bus stop, or even shift from bus to bicycle for the whole trip [40].

\subsection{Policy Implications}

To encourage bicycle use among older urban men and women in China, planning and public health policies should consider the gender differences in the effects of environmental factors. For both men and women, a safe environment at intersections and a positive attitude towards cycling may be effective, albeit to varying degrees. Therefore, we recommend two interventions: (1) enhancing safety for older cyclists at intersections, and (2) disseminating an active lifestyle oriented towards cycling activity. Safety issues at intersections may be a significant reason for older urban adults to make fewer cycling trips. Possible interventions include the improvement of road intersection design, traffic signals, and traffic management measures. Regarding attitudes, we recommended various initiatives (health-focused campaigns [60], public lectures, specialized websites, etc.) that have shown success in the "Ten Thousand Steps a Day" program [61].

For older women, less dense bus stops are associated with more cycling trips, implying that cycling interventions targeting older urban women may incorporate transit. However, this invention may harness bus use among older urban women. Therefore, the threshold effects and effective range of bus stop accessibility for both bicycle and bus use require further non-linear analysis.

For older men, interventions should consider the age structure and employment structure of a neighborhood and CBD accessibility. It may be effective to form vibrant neighborhoods with well-balanced age and employment structures. Locating more commercial establishments and service destinations adjacent to neighborhoods might also increase bicycle use among older urban men.

\section{Conclusions}

This study contributes to the land use-travel literature by disentangling the genderspecific relationship between environmental attributes and cycling activity among older urban adults, with data from Zhongshan, China. First, the study characterized built environment and social environment variables, together with sociodemographics and attitudes. Second, the study employed negative binomial regression to investigate the gender-specific environmental correlates of daily cycling trips among older urban adults. The results indicate that intersection density and attitudes towards different travel modes are significantly correlated to bicycle use among both genders in Zhongshan. For older urban men, the proportions of the aged or employed in a neighborhood and commercial accessibility show significance. However, bicycle use among older urban women is more correlated to transit service accessibility.

The findings facilitate the health promotion interventions and urban planning approach to accommodate older urban men and women equally from the perspective of built and social environment and attitudes. For both older urban men and women, we suggest creating a safe environment for cyclists at intersections and disseminating an active lifestyle relating to bicycle use. For older women, we suggest further discovering the threshold effects and the effective range of bus stop accessibility in favor of both bicycle and bus use. For older men, we suggest maintaining a relatively balanced age and employment structure in neighborhoods and providing abundant commercial and service destinations adjacent to residences.

This study has some limitations regarding data and method. First, the analysis employed cross-sectional data. The causal effects of different variables on bicycle use will require longitudinal data. Second, the study used linear modeling. Recent studies have begun to examine the non-linear relationships and threshold effects of environmental variables. The study yields several strengths. First, the study addressed the gender gap in 
the land use-travel literature. The findings provide nuanced policy implications for healthy aging. Second, the results indicate gender-specific environmental correlates of bicycle use among older urban adults in China. The findings will facilitate comparative studies among different contexts.

Author Contributions: L.W. led the survey design and manuscript preparation. Y.Z. conceived the research. X.L., X.C., J.W. and X.X. participated in data collection. C.L. and T.W. contributed to modeling. All authors have read and agreed to the published version of the manuscript.

Funding: This research was funded by the National Social Science Foundation of China (Grant No. 18BSH143).

Institutional Review Board Statement: Ethical review and approval were not required for the study on human participants in accordance with the local legislation and institutional requirements.

Informed Consent Statement: The participants provided their written informed consent to participate in this study.

Data Availability Statement: The data employed in this study are not readily available because they belong to ongoing projects (Grant No. 20692109900 and Grant No. 21692106700 of Shanghai Science and Technology Program, and Grant No. 2020-APTS-04 of APTSLAB).

Conflicts of Interest: The authors declare no conflict of interest.

\section{Appendix A}

Table A1. Descriptive statistics for variables.

\begin{tabular}{|c|c|c|c|c|c|c|c|c|c|}
\hline \multirow{2}{*}{ Variable } & \multirow{2}{*}{ Description } & \multicolumn{4}{|c|}{ Male (Sample Size $=648)$} & \multicolumn{4}{|c|}{ Female $($ Sample Size $=616)$} \\
\hline & & Mean & S. D. & Min. & Max. & Mean & S. D. & Min. & Max. \\
\hline \multicolumn{10}{|c|}{ Dependent variable } \\
\hline Frequency & Daily cycling trips, count & 0.37 & 0.91 & 0 & 8 & 0.18 & 0.63 & 0 & 5 \\
\hline \multicolumn{10}{|c|}{ Household variables } \\
\hline SIZE_1 & One member in a household, binary, $1=$ yes & 0.20 & 0.40 & 0 & 1 & 0.20 & 0.40 & 0 & 1 \\
\hline SIZE_2 & Two members in a household, binary, 1 = yes & 0.41 & 0.49 & 0 & 1 & 0.40 & 0.49 & 0 & 1 \\
\hline SIZE_2+ & Three or more members in a household, binary, $1=$ yes & 0.39 & 0.49 & 0 & 1 & 0.40 & 0.49 & 0 & 1 \\
\hline EMPLOYED & Number of household members employed, count & 0.70 & 0.96 & 0 & 5 & 0.71 & 0.93 & 0 & 4 \\
\hline H_HIGHINC & High household income (>6000 RMB/month), binary, 1 = yes & 0.19 & 0.40 & 0 & 1 & 0.21 & 0.41 & 0 & 1 \\
\hline H_MEDINC & Medium household income (2500-6000 RMB/month), binary, 1 = yes & 0.54 & 0.50 & 0 & 1 & 0.55 & 0.50 & 0 & 1 \\
\hline H_LOWINC & Low household income ( $<2500 \mathrm{RMB} /$ year), binary, 1 = yes & 0.26 & 0.44 & 0 & 1 & 0.24 & 0.43 & 0 & 1 \\
\hline \multicolumn{10}{|c|}{ Personal variables } \\
\hline AGE & Age in years, count & 67.62 & 6.68 & 60 & 95 & 67.25 & 6.52 & 60 & 93 \\
\hline P_HIGHINC & High personal income (>3000 RMB/month), binary, 1 = yes & 0.07 & 0.26 & 0 & 1 & 0.02 & 0.14 & 0 & 1 \\
\hline P_MEDINC & Medium personal income (1200-3000 RMB/month), binary, $1=$ yes & 0.74 & 0.44 & 0 & 1 & 0.76 & 0.43 & 0 & 1 \\
\hline P_LOWINC & Low personal income (<1200 RMB/month), binary, 1 = yes & 0.19 & 0.40 & 0 & 1 & 0.22 & 0.42 & 0 & 1 \\
\hline \multicolumn{10}{|c|}{ Attitudinal variables } \\
\hline FAV_BIKE & The respondent's favorite travel mode is bicycle, binary, 1 = yes & 0.16 & 0.37 & 0 & 1 & 0.09 & 0.28 & 0 & 1 \\
\hline FAV_WALK & The respondent's favorite travel mode is walking, binary, $1=$ yes & 0.27 & 0.45 & 0 & 1 & 0.39 & 0.49 & 0 & 1 \\
\hline FAV_EBIKE & The respondent's favorite travel mode is e-bike, binary, 1 = yes & 0.07 & 0.25 & 0 & 1 & 0.04 & 0.19 & 0 & 1 \\
\hline FAV_BUS & The respondent's favorite travel mode is bus, binary, $1=$ yes & 0.21 & 0.41 & 0 & 1 & 0.27 & 0.44 & 0 & 1 \\
\hline FAV_CAR & The respondent's favorite travel mode is car, binary, $1=$ yes & 0.19 & 0.37 & 0 & 1 & 0.21 & 0.40 & 0 & 1 \\
\hline \multicolumn{10}{|c|}{ Social environment variables } \\
\hline N_EMPLOY & Proportions of the employed in a neighborhood, continuous & 0.60 & 0.08 & 0.49 & 0.82 & 0.60 & 0.07 & 0.49 & 0.82 \\
\hline N_AGED & Proportions of the aged in a neighborhood, continuous & 0.17 & 0.07 & 0.04 & 0.29 & 0.18 & 0.07 & 0.04 & 0.29 \\
\hline N_AVGINC & Average monthly income of a neighborhood (in $100 \mathrm{RMB}$ ), continuous & 15.19 & 3.90 & 9.62 & 25.87 & 15.36 & 3.82 & 9.62 & 25.87 \\
\hline \multicolumn{10}{|c|}{ Built environment variables } \\
\hline DWELL_DEN & Dwelling units' density, 1000 dwelling units $/ \mathrm{km}^{2}$, continuous & 9.78 & 5.86 & 1.11 & 29.07 & 10.10 & 5.72 & 1.11 & 29.07 \\
\hline INTER_DEN & Intersection density, five intersections $/ \mathrm{km}^{2}$, continuous & 5.75 & 3.38 & 0.40 & 12.36 & 6.11 & 3.41 & 0.40 & 12.36 \\
\hline DIST_BUS & Distance between home and the closest bus stop, $\mathrm{km}$, continuous & 0.37 & 0.27 & 0.10 & 1.20 & 0.37 & 0.27 & 0.10 & 1.20 \\
\hline DIST_CBD & $\begin{array}{l}\text { Euclidean distance from the neighborhood centroid to the CBD, } \\
\text { in } \mathrm{km} \text {, continuous }\end{array}$ & 2.04 & 0.68 & 0.22 & 3.09 & 2.02 & 0.72 & 0.22 & 3.09 \\
\hline LAND_MIX & Entropy Index of land use mixture, continuous & 0.68 & 0.18 & 0.33 & 1.00 & 0.67 & 0.19 & 0.33 & 1.00 \\
\hline
\end{tabular}

Note: S. D. = Standard Deviation; Min. = minimum; Max. = maximum 


\section{References}

1. Wu, J.; Zhao, C.; Li, C.; Wang, T.; Wang, L.; Zhang, Y. Non-linear Relationships Between the Built Environment and Walking Frequency Among Older Adults in Zhongshan, China. Front. Public Health 2021, 9, 686144. [CrossRef] [PubMed]

2. World Health Organization. World Report on Ageing and Health; WHO: Geneva, Switzerland, 2015.

3. United Nations. World Population Ageing 2020-Highlights; Department of Economic and Social Affairs, United Nations: New York, NY, USA, 2020.

4. $\quad$ Nelson, M.E.; Rejeski, W.J.; Blair, S.N.; Duncan, P.W.; Judge, J.O.; King, A.C.; Macera, C.A.; Castaneda-Sceppa, C. Physical activity and public health in older adults: Recommendation from the American College of Sports Medicine and the American Heart Association. Med. Sci. Sports Exerc. 2007, 39, 1435. [CrossRef] [PubMed]

5. Keysor, J.J. Does late-life physical activity or exercise prevent or minimize disablement? A critical review of the scientific evidence. Am. J. Prev. Med. 2003, 25, 129-136. [CrossRef]

6. De Hartog, J.J.; Boogaard, H.; Nijland, H.; Hoek, G. Do the Health Benefits of Cycling Outweigh the Risks? Environ. Health Perspect. 2010, 118, 1109-1116. [CrossRef] [PubMed]

7. Drca, N.; Wolk, A.; Jensen-Urstad, M.; Larsson, S.C. Physical activity is associated with a reduced risk of atrial fibrillation in middle-aged and elderly women. Heart 2015, 101, 1627-1630. [CrossRef] [PubMed]

8. Macaluso, A.; Young, A.; Gibb, K.S.; Rowe, D.A.; De Vito, G. Cycling as a novel approach to resistance training increases muscle strength, power, and selected functional abilities in healthy older women. J. Appl. Physiol. 2003, 95, 2544-2553. [CrossRef]

9. Winters, M.; Sims-Gould, J.; Franke, T.; McKay, H. "I grew up on a bike": Cycling and older adults. J. Transp. Health 2015, 2, 58-67. [CrossRef]

10. Handy, S.; van Wee, B.; Kroesen, M. Promoting Cycling for Transport: Research Needs and Challenges. Transp. Rev. 2014, 34, 4-24. [CrossRef]

11. Metz, D. Transport policy for an ageing population. Transp. Rev. 2003, 23, 375-386. [CrossRef]

12. Fraser, S.D.S.; Lock, K. Cycling for transport and public health: A systematic review of the effect of the environment on cycling. Eur. J. Public Health 2011, 21, 738-743. [CrossRef]

13. Handy, S.L.; Boarnet, M.G.; Ewing, R.; Killingsworth, R.E. How the built environment affects physical activity: Views from urban planning. Am. J. Prev. Med. 2002, 23, 64-73. [CrossRef]

14. Saelens, B.E.; Sallis, J.F.; Black, J.B.; Chen, D. Neighborhood-based differences in physical activity: An environment scale evaluation. Am. J. Public Health 2003, 93, 1552-1558. [CrossRef]

15. Cao, X.Y.; Mokhtarian, P.L.; Handy, S.L. Neighborhood Design and the Accessibility of the Elderly: An Empirical Analysis in Northern California. Int. J. Sustain. Transp. 2010, 4, 347-371. [CrossRef]

16. Brownson, R.C.; Hoehner, C.M.; Day, K.; Forsyth, A.; Sallis, J.F. Measuring the Built Environment for Physical Activity State of the Science. Am. J. Prev. Med. 2009, 36, S99-S123. [CrossRef] [PubMed]

17. McCormack, G.R.; Shiell, A. In search of causality: A systematic review of the relationship between the built environment and physical activity among adults. Int. J. Behav. Nutr. Phys. Act. 2011, 8, 125. [CrossRef] [PubMed]

18. Van Cauwenberg, J.; De Bourdeaudhuij, I.; De Meester, F.; Van Dyck, D.; Salmon, J.; Clarys, P.; Deforche, B. Relationship between the physical environment and physical activity in older adults: A systematic review. Health Place 2011, 17, 458-469. [CrossRef]

19. Saelens, B.E.; Handy, S.L. Built environment correlates of walking: A review. Med. Sci. Sports Exerc. 2008, 40, S550. [CrossRef]

20. Willis, D.P.; Manaugh, K.; El-Geneidy, A. Cycling Under Influence: Summarizing the Influence of Perceptions, Attitudes, Habits, and Social Environments on Cycling for Transportation. Int. J. Sustain. Transp. 2015, 9, 565-579. [CrossRef]

21. Heinen, E.; van Wee, B.; Maat, K. Commuting by Bicycle: An Overview of the Literature. Transp. Rev. 2010, 30, 59-96. [CrossRef]

22. Roof, K.; Oleru, N. Public health: Seattle and King County's push for the built environment. J. Environ. Health $2008,71,24-27$.

23. Srinivasan, S.; O'Fallon, L.R.; Dearry, A. Creating healthy communities, healthy homes, healthy people: Initiating a research agenda on the built environment and public health. Am. J. Public Health 2003, 93, 1446-1450. [CrossRef]

24. Van Cauwenberg, J.; Clarys, P.; De Bourdeaudhuij, I.; Van Holle, V.; Verte, D.; De Witte, N.; De Donder, L.; Buffel, T.; Dury, S.; Deforche, B. Physical environmental factors related to walking and cycling in older adults: The Belgian aging studies. BMC Public Health 2012, 12, 142. [CrossRef] [PubMed]

25. Zhang, Y.; Yang, X.G.; Li, Y.; Liu, Q.X.; Li, C.Y. Household, Personal and Environmental Correlates of Rural Elderly's Cycling Activity: Evidence from Zhongshan Metropolitan Area, China. Sustainability 2014, 6, 3599-3614. [CrossRef]

26. Verhoeven, H.; Simons, D.; Van Dyck, D.; Van Cauwenberg, J.; Clarys, P.; De Bourdeaudhuij, I.; de Geus, B.; Vandelanotte, C.; Deforche, B. Psychosocial and Environmental Correlates of Walking, Cycling, Public Transport and Passive Transport to Various Destinations in Flemish Older Adolescents. PLoS ONE 2016, 11, e0147128. [CrossRef]

27. Ji, Y.J.; Fan, Y.L.; Ermagun, A.; Cao, X.N.; Wang, W.; Das, K. Public bicycle as a feeder mode to rail transit in China: The role of gender, age, income, trip purpose, and bicycle theft experience. Int. J. Sustain. Transp. 2017, 11, 308-317. [CrossRef]

28. Ewing, R.; Cervero, R. Travel and the built environment: A meta-analysis. J. Am. Plan. Assoc. 2010, 76, 265-294. [CrossRef]

29. Li, Z.; Wang, W.; Yang, C.; Ding, H. Bicycle mode share in China: A city-level analysis of long term trends. Transportation 2017, 44, 773-788. [CrossRef]

30. Kemperman, A.; Timmermans, H. Influences of Built Environment on Walking and Cycling by Latent Segments of Aging Population. Transp. Res. Rec. 2009, 2134, 1-9. [CrossRef] 
31. Leger, S.J.; Dean, J.L.; Edge, S.; Casello, J.M. "If I had a regular bicycle, I wouldn't be out riding anymore": Perspectives on the potential of e-bikes to support active living and independent mobility among older adults in Waterloo, Canada. Transp. Res. Part A-Policy Pract. 2019, 123, 240-254. [CrossRef]

32. Mertens, L.; Van Dyck, D.; Deforche, B.; De Bourdeaudhuij, I.; Brondeel, R.; Van Cauwenberg, J. Individual, social, and physical environmental factors related to changes in walking and cycling for transport among older adults: A longitudinal study. Health Place 2019, 55, 120-127. [CrossRef]

33. Grimes, A.; Chrisman, M.; Lightner, J. Barriers and Motivators of Bicycling by Gender Among Older Adult Bicyclists in the Midwest. Health Educ. Behav. 2020, 47, 67-77. [CrossRef]

34. Van Cauwenberg, J.; De Bourdeaudhuij, I.; Clarys, P.; De Geus, B.; Deforche, B. Older adults' environmental preferences for transportation cycling. J. Transp. Health 2019, 13, 185-199. [CrossRef]

35. Moudon, A.V.; Lee, C.; Cheadle, A.D.; Collier, C.W.; Johnson, D.; Schmid, T.L.; Weather, R.D. Cycling and the built environment, a US perspective. Transp. Res. Part. D-Transport. Environ. 2005, 10, 245-261. [CrossRef]

36. Siman-Tov, M.; Jaffe, D.H.; Peleg, K.; Israel Trauma, G. Bicycle injuries: A matter of mechanism and age. Accid. Anal. Prev. 2012, 44, 135-139. [CrossRef] [PubMed]

37. Prins, R.G.; Pierik, F.; Etman, A.; Sterkenburg, R.P.; Kamphuis, C.B.M.; van Lenthe, F.J. How many walking and cycling trips made by elderly are beyond commonly used buffer sizes: Results from a GPS study. Health Place 2014, 27, 127-133. [CrossRef]

38. Van Cauwenberg, J.; De Bourdeaudhuij, I.; Clarys, P.; de Geus, B.; Deforche, B. Environmental Preferences for Transportation Cycling Among Older Adults: An Experiment with Manipulated Photographs. J. Transport. Health 2018, 9, S4. [CrossRef]

39. Barnett, E.; Casper, M. A definition of "social environment". Am. J. Public Health 2001, 91, 465.

40. Wang, L.; Zhao, C.; Liu, X.; Chen, X.; Li, C.; Wang, T.; Wu, J.; Zhang, Y. Non-Linear Effects of the Built Environment and Social Environment on Bus Use among Older Adults in China: An Application of the XGBoost Model. Int. J. Environ. Res. Public Health 2021, 18, 9592. [CrossRef]

41. Lee, Y.-S. Gender Differences in Physical Activity and Walking Among Older Adults. J. Women Aging 2005, 17, 55-70. [CrossRef]

42. Gallagher, N.A.; Clarke, P.J.; Gretebeck, K.A. Gender Differences in Neighborhood Walking in Older Adults. J. Aging Health 2014, 26, 1280-1300. [CrossRef]

43. Zhang, Y.; Chen, L.; Zhu, W.; Liu, X.; Chen, D. Relationship between physical activity and environment in Shanghai, China: Analysis and evaluation in adults aged 45-80. Med. Sport 2011, 64, 269-284.

44. Zhang, Y.; Li, C.; Ding, C.; Zhao, C.; Huang, J. The Built Environment and the Frequency of Cycling Trips by Urban Elderly: Insights from Zhongshan, China. J. Asian Archit. Build. Eng. 2018, 15, 511-518. [CrossRef]

45. National Health and Family Planning Commission of China. Promotion of Healthy Living Style in China; National Health and Family Planning Commission of China (NHFPC): Beijing, China, 2007.

46. Zhang, Y.; He, Q.; Wu, W.; Li, C.Y. Public transport use among the urban and rural elderly in China: Effects of personal, attitudinal, household, social-environment and built-environment factors. J. Transp. Land Use 2018, 11, 701-719. [CrossRef]

47. Zhang, Y.; Li, Y.; Yang, X.; Liu, Q.; Li, C. Built Environment and Household Electric Bike Ownership. Transp. Res. Rec. J. Transp. Res. Board 2013, 2387, 102-111. [CrossRef]

48. Zhang, Y.; Li, Y.; Liu, Q.; Li, C. The Built Environment and Walking Activity of the Elderly: An Empirical Analysis in the Zhongshan Metropolitan Area, China. Sustainability 2014, 6, 1076-1092. [CrossRef]

49. National Bureau of Statistics of China. China Statistical Yearbook 2012; China Statistics Press: Beijing, China, 2012.

50. Zhongshan Municipal Bureau of Urban Planning. Zhongshan Household Travel Survey; Zhongshan Municipal Bureau of Urban Planning: Zhongshan, China, 2012.

51. Martinez, L.M.; Viegas, J.M.; Silva, E.A. A traffic analysis zone definition: A new methodology and algorithm. Transportation 2009, 36, 581-599. [CrossRef]

52. Ding, C.; Wang, D.; Liu, C.; Zhang, Y.; Yang, J. Exploring the influence of built environment on travel mode choice considering the mediating effects of car ownership and travel distance. Transp. Res. Part A-Policy Pract. 2017, 100, 65-80. [CrossRef]

53. Kockelman, K.M. Travel behavior as function of accessibility, land use mixing, and land use balance: Evidence from San Francisco Bay Area. Transp. Res. Rec. J. Transp. Res. Board 1997, 1607, 116-125. [CrossRef]

54. Ministry of Housing and Urban-Rural Development of the People's Republic of China. Code for Urban Land Use Classes and Standards of Planning Construction Land (GB50137-2011); Ministry of Housing and Urban-Rural Development of the People's Republic of China: Beijing, China, 2011. (In Chinese)

55. Zhongshan Municipal Bureau of Urban Planning. Zhongshan Transportation Development Planning; Zhongshan Municipal Bureau of Urban Planning: Zhongshan, China, 2012.

56. Liu, P.; Marker, S. Evaluation of contributory factors' effects on bicycle-car crash risk at signalized intersections. J. Transp. Saf. Secur. 2020, 12, 82-93. [CrossRef]

57. Congiu, T.; Sotgiu, G.; Castiglia, P.; Azara, A.; Piana, A.; Saderi, L.; Dettori, M. Built Environment Features and Pedestrian Accidents: An Italian Retrospective Study. Sustainability 2019, 11, 1064. [CrossRef]

58. Ball, K.; Jeffery, R.W.; Abbott, G.; McNaughton, S.A.; Crawford, D. Is healthy behavior contagious: Associations of social norms with physical activity and healthy eating. Int. J. Behav. Nutr. Phys. Act. 2010, 7, 86. [CrossRef] [PubMed] 
59. Wang, W.; Zhang, Y.; Zhao, C.; Liu, X.; Chen, X.; Li, C.; Wang, T.; Wu, J.; Wang, L. Nonlinear Associations of the Built Environment with Cycling Frequency among Older Adults in Zhongshan, China. Int. J. Environ. Res. Public Health 2021, 18, 10723. [CrossRef] [PubMed]

60. Gamble, T.; Walker, I.; Laketa, A. Bicycling campaigns promoting health versus campaigns promoting safety: A randomized controlled online study of 'dangerization'. J. Transp. Health 2015, 2, 369-378. [CrossRef]

61. Yang, Z. Behavior and health. Chin. J. Behav. Med. Brain Sci. 2009, 18, 61-63. [CrossRef] 